\title{
An investigation on effects of flow parameters on unsteady MHD flow in a porous medium bounded by parallel porous vertical plates
}

\author{
Ronald Nyabuto ${ }^{1,}$ Jeconiah Abonyo Okelo ${ }^{2}$ and Johana Kibet Sigey ${ }^{3}$
}

Department of mathematical sciences, Jomo Kenyatta University of agriculture and technology, Kenya

\begin{abstract}
The effects of flow parameter in a porous medium confined to parallel, vertical, porous plates on unstable viscous fluid were investigated in this research. The fluid was applied perpendicularly in the positive y-direction to a consistent magnetic field. The results are discussed and displayed in graphical order using explicit, minimal discrepancies approximations on the effects of the Darcy numbers, suction variables, Prandtl and grash numbers on speed profiles and temperature distribution.
\end{abstract}

Key terms: Incompressible, Porous medium, Unsteady.

\section{INTRODUCTION}

Flows through porous media are very much prevalent in nature due to their wide applications in both science and engineering. A considerable attention has been drawn on this area through varied investigations. This is aimed at having a better understanding on the fundamental mechanism encountered on both warm and flow processes in porous media. Flows through porous media are widely encountered in the study of underground water resources, seepage of water in riverbeds. Energy transmission between the electromagnetic field and the fluid occurs in MHD flows. This depends, however, on the intensity of the magnetic field applied.

\section{LITERATURE REVIEW}

Several porous media studies have been carried out with MHD flows. Substantial work on viscous streams in porous media has been carried out with different geometries. An overview of the magnetic effects of free convection and mass transmission through porous media and continuous suction and warm stream was one of the most recent researches for these subjects. Medium porosity influences the speed and temperature distribution. With a higher permeability parameter, both profiles are increased. Incompressible viscous fluid, a transient non-convective convective fluid, is investigated [9]. This study was carried out. The governing equations were precisely solved using the finite differential method. You can find that the amount of Prandtl increases with time. Free convection and mass transmission of hydro-magnetic objects, heating, thermal dispersion, hall electricity and heat source was studied [6]. In the nonlinear differential partial equations dominated by disturbance technology, the Hall parameter was included.

Manuscript received: 24 August 2020

Manuscript received in revised form: 21 September 2020

Manuscript accepted: 07 October 2020

Manuscript Available online: 15 October 2020
The primary friction of the skin increased. Higher Schmidt-Grash of numbers [5] has also carried out a porous, magnetically field-induced platform for an unstable flow transfer analysis from a viscous, incompressible boundary. The term viscoso was used and solved by explicit finite difference approximations in the governing equations. The speed profile and temperature profiles were improved with increased Grashof numbers and reduced Grashof numbers. Magnets have been reduced induction. Study is carried out using semi-analytic approaches to the MHD boundary layer using a non-linear stretch sheet in a porous medium [8]. The Temperature and Concentration Profiles were decreased respectively by Prandtl and Schimidt. In the presence of porous medium with a chemical reaction, Loganathan and Sivapoornapriya studied unstable natural convective flow through an instinctive, semi-medical vertical plate. On a porous vertical plate [4] of unstable free convection and mass transmission flow, on the other hand, a numerical analysis was performed. In a rotating device with a current source in hall and a porous vertical layer, an open, MHD convective thermal convector and transfer flux analysis was performed [10]. By using a finite difference method, they precisely solved their partial differential equation and found that the temperature rises with an increase in time, in Grashof and in Hall. It has not been thoroughly explored and the controlling equations of each studies are studied in detail. The impact of flow parameters on unstable, porous, and intermediated flow. The goal of this study was to fully define the effect on MHD by vertical infinite pore plates in transverted magnetic fields of the flow parameter such as suction parameters, prandtl, Darcy, and Grashof numbers.

\section{PROBLEM FORMULATION}

An electrically conducive, viscous, incompressible fluid moving an infinite vertical, porous layer is called an incessant free-convection flow. Perpendicular to the plate is applied a uniform magnetic field $\mathrm{B}$ in the positive $\mathrm{Y}$-direction. The $\mathrm{x}$ axis is taken up the infinite porous vertical plate, as seen in figure (1), and it is normal to the platform to the y axis. The plates were assumed to be porous, electrically non-conducting $\&$ very long in the $\mathrm{x}-\mathrm{z}$ direction in this problem. In addition, the fluid was regarded to be incompressible with constant density and small Reynolds number. Effects of Hall current were ignored since a weak magnetic field was applied hence The Ohm's law is negligible. 


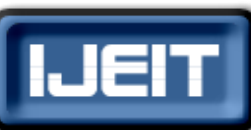

ISSN: 2277-3754

ISO 9001:2008 Certified

International Journal of Engineering and Innovative Technology (IJEIT)

$\mathrm{X}$-axis

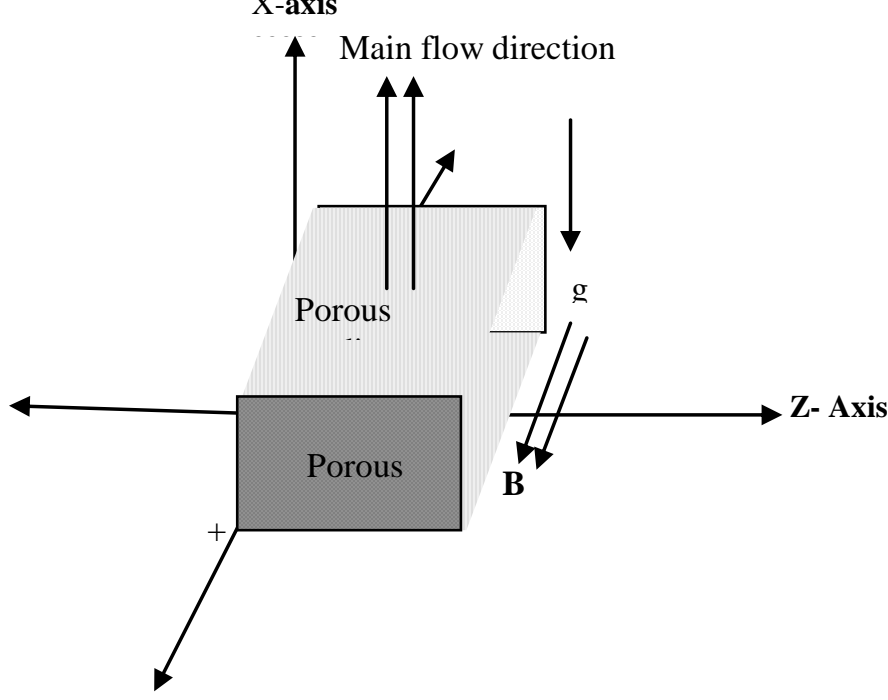

Fig.1. Configuration of geometry

The governing equations of the problem are expressed as follow; the continuity equation is simplified to

$\frac{\partial v}{\partial y}=0$

Integrating the equation (1) gives

$V=-v_{0}$

The momentum equation is expressed as

$\rho\left[\frac{\partial \bar{v}}{\partial \mathrm{t}}+(\overline{\mathrm{v}} \cdot \nabla) \overline{\mathrm{v}}\right]=-\nabla \mathrm{P}+\mu \nabla^{2} \overline{\mathrm{v}}-\rho \mathrm{g}-\frac{\partial \mathrm{u}}{\mathrm{K}_{\mathrm{p}}}$

The equation of continuity coupled with the simplified momentum equation into $\mathrm{x}$-component and w-component. The $\mathrm{x}$-component is

$\rho\left(\frac{\partial u}{\partial t}+u \frac{\partial u}{\partial x}+v \frac{\partial u}{\partial y}\right)=-\frac{\partial p}{\partial x}+\mu \frac{\partial^{2} u}{\partial y^{2}}-\rho g-\frac{\partial u}{K_{p}}$

(4)

Equation (4) simplifies to

$\frac{\partial \mathrm{u}}{\partial \mathrm{t}}=\theta \frac{\partial^{2} \mathrm{u}}{\partial \mathrm{y}^{2}}+v_{0} \frac{\partial \mathrm{u}}{\partial y}+\beta g\left(\mathrm{~T}_{2}-\mathrm{T}_{1}\right)-\frac{\partial}{\mathrm{K}_{\mathrm{p}}} \mathrm{u}$

(5)

The z-component is

$\frac{\partial w}{\partial t}=\theta \frac{\partial^{2} w}{\partial y^{2}}+v_{0} \frac{\partial w}{\partial y}-\frac{\partial w}{K_{p}}$

Energy equation is fundamentally established from the first law of thermodynamics and is simplified as

$\rho C_{p} \frac{D_{T}}{D_{t}}=\nabla \cdot(K V \cdot T)+\mu \varphi$

Upholding the considerations made in this problem, the equation (7) simplifies to

$$
\mathrm{\rho C}_{\mathrm{p}}\left(\frac{\partial \mathrm{T}}{\partial \mathrm{t}}-\mathrm{v}_{0} \frac{\partial \mathrm{T}}{\partial \mathrm{y}}\right)=\mathrm{k} \frac{\partial^{2} \mathrm{~T}}{\partial y^{2}}+\mu\left[\left(\frac{\partial u}{\partial y}\right)^{2}+\left(\frac{\partial w}{\partial y}\right)^{2}\right]
$$

$\mathrm{t} \leq 0, \mathrm{u}=0, w=0, \mathrm{~T}=0$ at $\mathrm{y}=0$

$\mathrm{t}>0, \quad \mathrm{u}=0, \mathrm{w}=0$ and $\mathrm{T}=\mathrm{T}_{0} \quad \forall \quad \mathrm{y}(9)$

The following non-dimensional variables are used in momentum and energy equations so as to reduce their complexity.

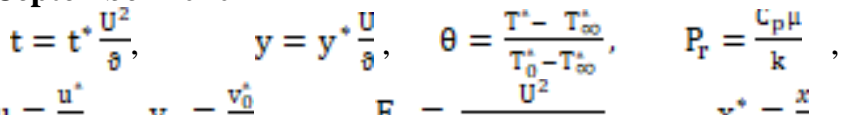

$\mathrm{u}=\frac{\mathrm{u}^{\mathrm{s}}}{\mathrm{U}}, \quad \mathrm{v}_{0}=\frac{\mathrm{w}_{0}^{\mathrm{a}}}{\mathrm{U}}, \quad \mathrm{E}_{\mathrm{c}}=\frac{\mathrm{U}^{2}}{\mathrm{C}_{\mathrm{p}}\left[\mathrm{T}_{\mathrm{W}}^{*}-\mathrm{T}_{\mathrm{w}}^{*}\right)}, \quad \mathrm{x}^{*}=\frac{x}{\mathbb{d}}$

Consequently, the $\mathrm{x}$-component equation (5) is reduced into

$$
\frac{\partial \mathrm{u}}{\partial \mathrm{t}}=\frac{\partial^{2} \mathrm{u}}{\partial \mathrm{y}^{2}}+v_{0} \frac{\partial \mathrm{u}}{\partial \mathrm{y}}+\beta \mathrm{g} \Delta \mathrm{T} \frac{\partial}{\mathrm{U}^{3}}-\left(\frac{\partial}{\mathrm{K}_{\mathrm{p}}}\right) \frac{\partial}{\mathrm{U}^{2}} \mathrm{u}
$$

Equation (10) on using non dimensional numbers simplifies to

$$
\frac{\partial u}{\partial t}=\frac{\partial^{2} \mathrm{u}}{\partial y^{2}}+v_{0} \frac{\partial u}{\partial y}+\mathrm{Gr} \theta-\frac{1}{D a} \mathrm{u}
$$

Similarly, the z-component of momentum equation becomes $\frac{\partial w}{\partial t}=\frac{\partial^{2} w}{\partial y^{2}}+v_{0} \frac{\partial w}{\partial y}-\frac{\partial^{2}}{K_{p} U^{2}} w$

Equation (12) on using non-dimensional numbers collapses to

$\frac{\partial w}{\partial t}=\frac{\partial^{2} w}{\partial y^{2}}+v_{0} \frac{\partial w}{\partial y}-\frac{1}{D a} u$

On the other hand, the energy equation (8) is dimensionilized to become

$\frac{\partial \theta}{\partial \mathrm{t}}-\mathrm{v}_{0} \frac{\partial \theta}{\partial y}=\frac{\mathrm{k}}{\mu \mathrm{C}_{\mathrm{p}}} \cdot \frac{\partial^{2} \theta}{\partial y^{2}}+\frac{\mathrm{U}^{2}}{\mathrm{C}_{\mathrm{p}} \Delta \mathrm{T}} \cdot\left[\left(\frac{\partial \mathrm{u}}{\partial \mathrm{y}}\right)^{2}+\left(\frac{\partial \mathrm{w}}{\partial y}\right)^{2}\right]$

Equation (14) simplifies to

$$
\frac{\partial \theta}{\partial t}-v_{0} \frac{\partial \theta}{\partial y}=\frac{1}{\mathrm{Pr}_{\mathrm{r}}} \cdot \frac{\partial^{2} \theta}{\partial y^{2}}+\mathrm{Ec} \cdot\left[\left(\frac{\partial u}{\partial y}\right)^{2}+\left(\frac{\partial w}{\partial y}\right)^{2}\right]
$$

The boundary conditions applied in this flow problem are;

$\mathrm{t} \leq 0, \mathrm{u}=1, \mathrm{w}=0, \theta=1$ at $\mathrm{y}=0$

$\mathrm{t}>0, \mathrm{u}=0, \mathrm{w}=0, \quad \theta=0$ for all $\mathrm{y}$

\section{METHOD OF SOLUTION}

The partial differential equations (11), (13) and (15) are numerically solved using an explicit scheme for finite differences along with original and boundary conditions (9) and (16). In this scheme, a partial differential equation will be replaced by a discreet approximation which leads to a number of algebraic equations solved for discrete unknown values. In order to use this method, partial derivatives in the first and second order are replaced by its corresponding Taylors series.

Momentum in $\mathrm{x}$-axis direction

$\left(\frac{\mathrm{U}_{\mathrm{i}}^{\mathrm{i}+1}-\mathrm{U}_{\mathrm{i}}^{\mathrm{i}}}{\Delta \mathrm{t}}\right)=\left(\frac{\mathrm{U}_{\mathrm{i}-1}^{\mathrm{i}}-2 \mathrm{U}_{\mathrm{i}}^{\mathrm{i}}+\mathrm{U}_{\mathrm{i}+1}^{\mathrm{i}}}{[\Delta \mathrm{dy})^{2}}\right)+v_{0}\left(\frac{\mathrm{U}_{\mathrm{i}+1}^{\mathrm{i}}-\mathrm{U}_{\mathrm{i}}^{\mathrm{i}}}{\Delta y}\right)+\mathrm{Gr}_{\mathrm{r}} \theta_{\mathrm{i}}^{\mathrm{i}}-\frac{1}{\mathrm{Da}} \mathrm{U}_{\mathrm{i}}^{\mathrm{i}}$

Momentum in $\mathrm{z}$ - axis direction

$\left(\frac{W_{i}^{i}+1-W_{i}^{i}}{\Delta t}\right)=\left(\frac{W_{i-1}^{i}-2 W_{i}^{i}+W_{i+1}^{i}}{\Delta \Delta y)^{2}}\right)+v_{0}\left(\frac{W_{i+1}^{i}-W_{i}^{i}}{\Delta y}\right)-\frac{1}{D a} U_{i}^{i}$ 


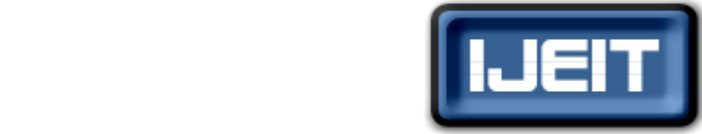

ISSN: 2277-3754

ISO 9001:2008 Certified

International Journal of Engineering and Innovative Technology (IJEIT)

Volume 10, Issue 3, September 2020

The energy equation in finite difference approximation is written as

$$
\left(\frac{\theta_{i}^{i+1}-\theta_{i}^{i}}{\Delta t}\right)=\frac{1}{\operatorname{Pr}^{2}} \cdot\left(\frac{\theta_{i-1}^{i}-2 \theta_{i}^{i}+\theta_{i+1}^{i}}{\Delta \Delta y)^{2}}\right)+v_{0}\left(\frac{\theta_{i+1}^{i}-\theta_{i}^{i}}{\Delta y}\right)+E c\left[\left(\frac{v_{i+1}^{i}-v_{i}^{i}}{\Delta y}\right)^{2}+\left(\frac{W_{i+1}^{i}-W_{i}^{i}}{\Delta y}\right)^{2}\right]
$$

$$
\left.\begin{array}{c}
\mathrm{t}=0, \mathrm{U}_{\mathrm{i}}^{0}=0, \mathrm{~W}_{\mathrm{i}}^{0}=0, \theta_{\mathrm{i}}^{0}=0 \\
\mathrm{t}>0, \mathrm{U}_{-1}^{\mathrm{j}}=\mathbb{1}_{s} \mathrm{~W}_{-1}^{\mathrm{j}}=0 \quad \theta_{-1}^{\mathrm{j}}=1 \\
\mathrm{U}_{1}^{\mathrm{j}}=0, \mathrm{~W}_{1}^{\mathrm{j}}=0 \theta_{1}^{\mathrm{j}}=0
\end{array}\right\}
$$

\section{RESULTS AND DISCUSSIONS}

During the investigation of the flow problem, Darcy number, Prandtl number, Grashof number and Suction parameter were generated. The effects of each of these parameters on the flow behavior in regard to their contribution to velocity field and temperature distribution are discussed in detail as follows;

\section{A. Effect of Darcy Number}

Darcy number relates fluid velocity to ability of the porous material to allow a liquid or a gas through its pores. When there is a decrease in pore size of the porous medium resistance to the flow is increased.

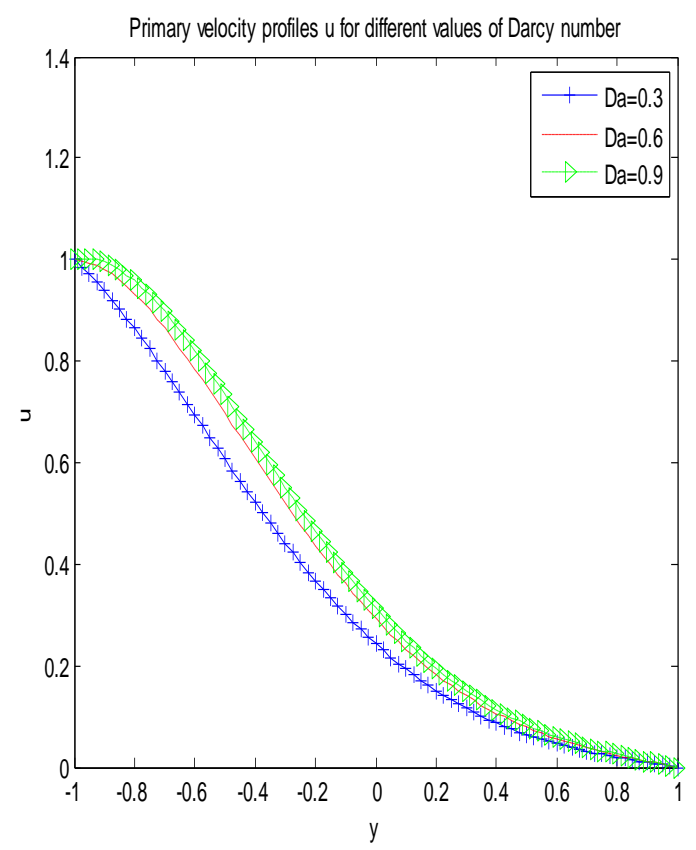

Fig.1.Impact of Darcy Number on primary velocity

For this reason, an increase in Darcy number of the medium tends to speed up the fluid velocities in both primary and secondary directions. It is also found that a raise in Darcy number promotes fluid velocity but leads to a minimal effect on temperature distribution as shown by figure 3 .

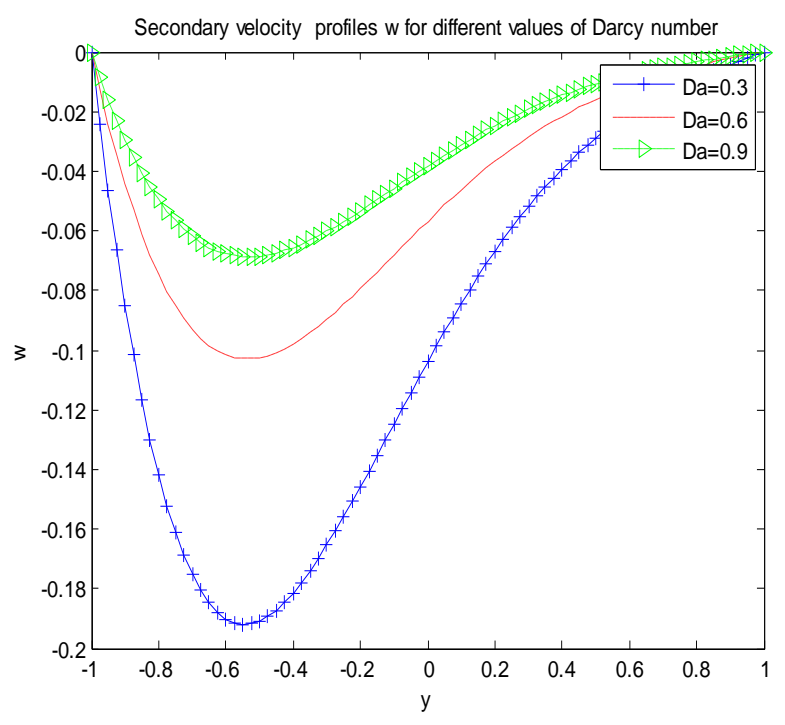

Fig.2. Impact of Darcy number on secondary velocity

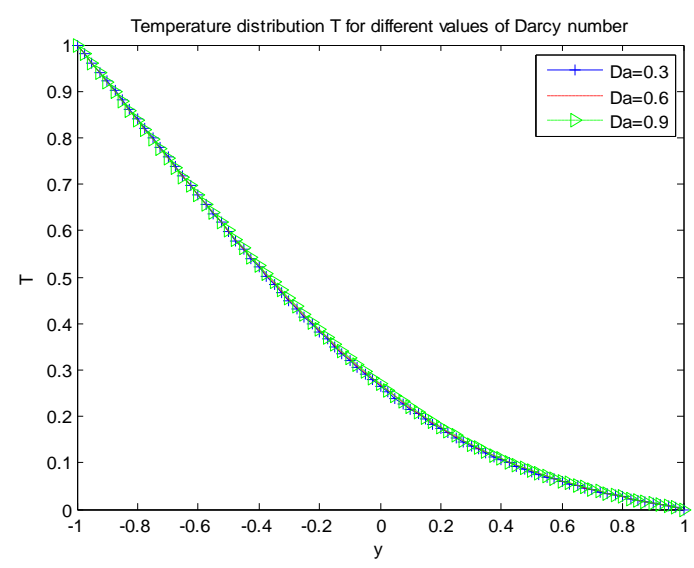

Fig.3.Impact of Darcy Number on temperature distribution

\section{B. Effect of Grashof Number}

This is in line with the physical situation, since an increase in the amount of Grashof means an increase in the buoyancy force. This force tends in both primary and secondary directions to speed up the fluid flow.

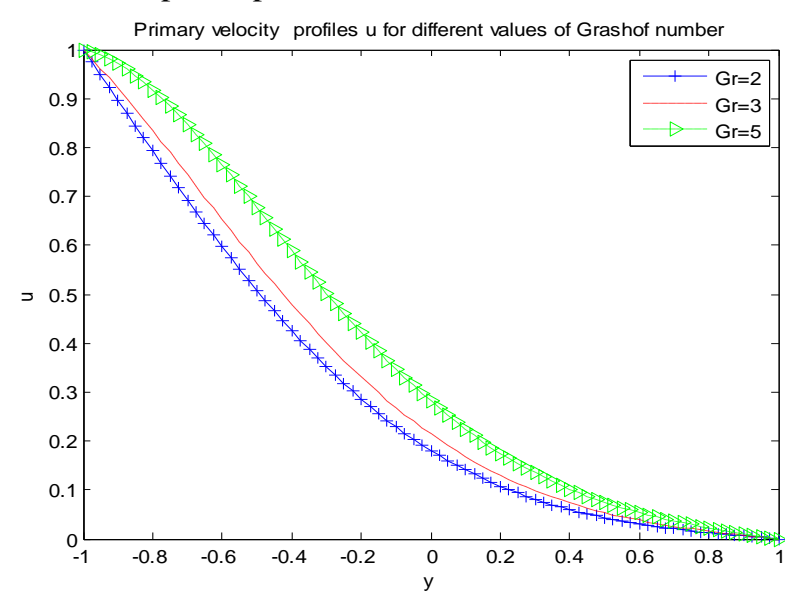

Fig.4. Impact of Grashof Number on Primary Velocity 
ISSN: 2277-3754

ISO 9001:2008 Certified

International Journal of Engineering and Innovative Technology (IJEIT)

Volume 10, Issue 3, September 2020

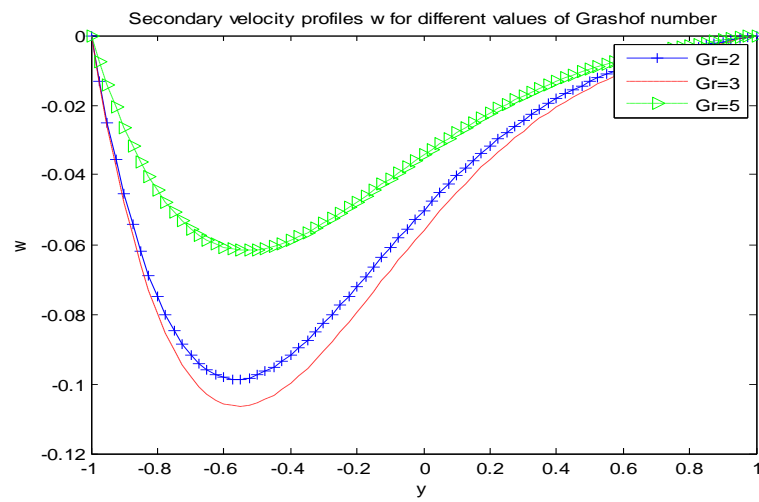

Fig.5. Impact of Grashof Number on Secondary Velocity

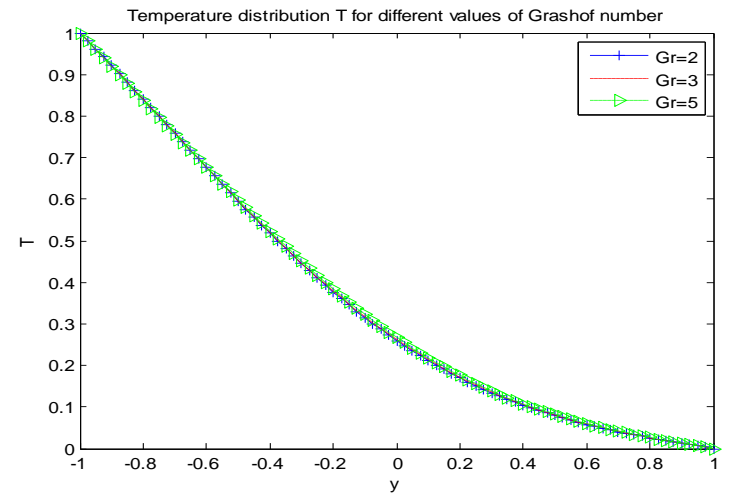

Fig.6. Impact of Grashof Number on Temperature

On the other hand, it has been found that Grashof number has a small effect on the increase in temperature of the fluid flow as indicated by figure 6 .

\section{Effect of Suction Parameter}

It was also found that an increase in suction cause a reduction in primary velocity as shown in figures 7 with an increase in secondary velocity as indicated in figures 8 . On the other hand, temperature of the fluid is found to decrease with increasing suction parameter as depicted by figure 9 . This is so due to the fact that an increase in suction implies more fluid is pressed into the fluid throughout the plate which there by reduce temperature field.

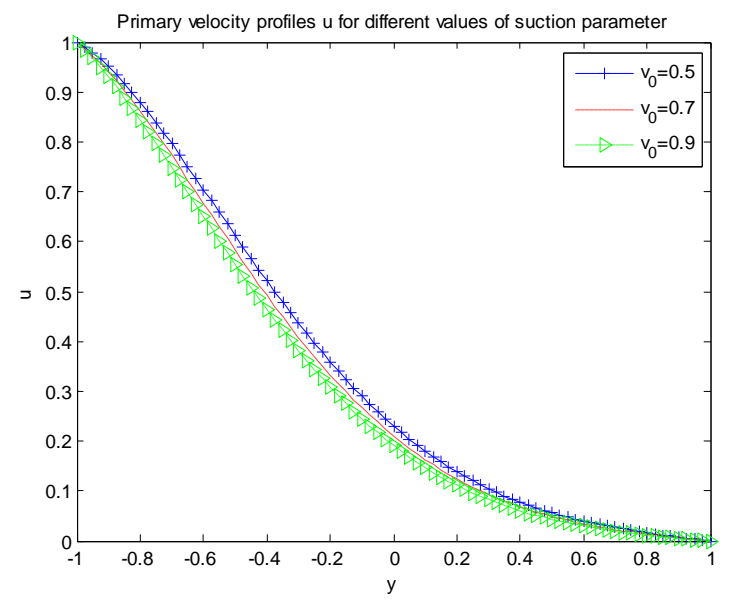

Fig.7. Suction's Impact on Primary Velocity

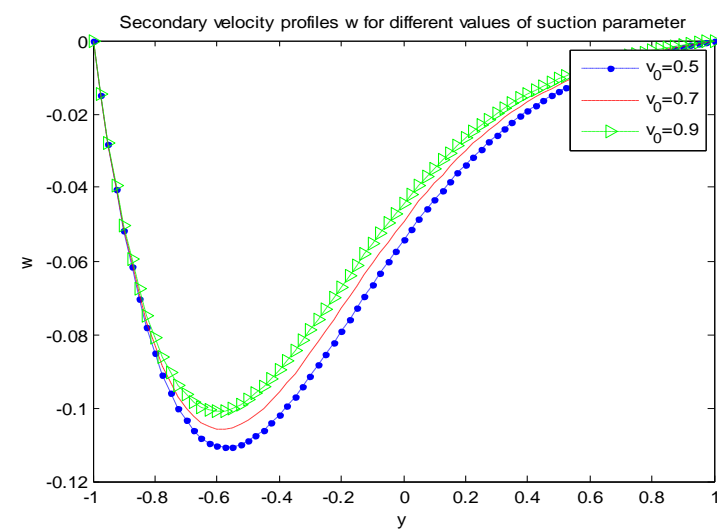

Fig.8. Impact of Suction on Secondary Velocity

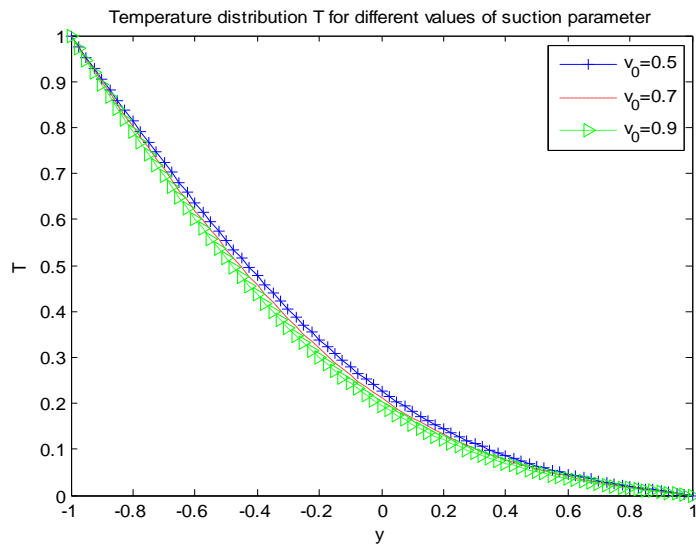

Fig.9. Impact of Suction on Temperature

\section{Effect of Prandtl Number}

Prandtl number represents a measure of the relative viscosity strength to thermal fluid diffusivity. In this analysis, we chose standard Prandtl values for air, salt water, and clean water at $0.71,1.0$ and 7.0 for their physical significance, according to the definition [7]. Figures 10 an 11 show that an raise in the amount of Prandtl results in a reduce in the primary speed and a rise in the secondary speed. It is clearly seen from these figures that the degree of primary and secondary velocities for air ( ) is greater than that of water ( )) everywhere in the flow field.

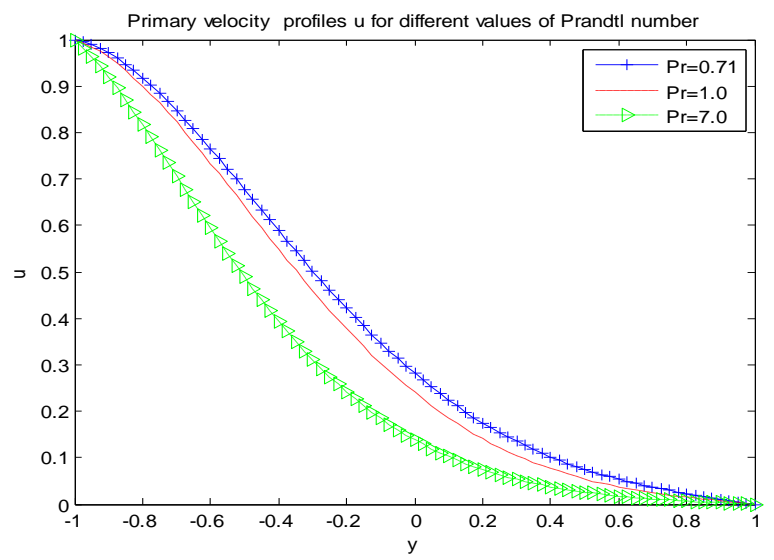

Fig.10.Impact of Prandtl Number on Primary Velocity 


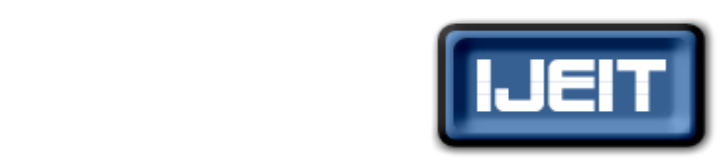

\section{ISSN: 2277-3754 \\ ISO 9001:2008 Certified \\ International Journal of Engineering and Innovative Technology (IJEIT) \\ Volume 10, Issue 3, September 2020}

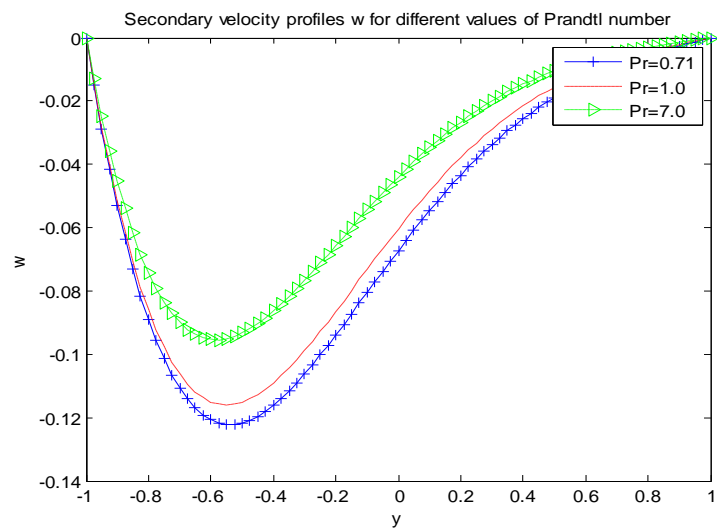

Fig.11.Impact of Prandtl Number on Secondary Velocity

Furthermore, an increase in Prandtl was found to contribute to a decrease in the fluid's temperature distribution as seen in Figures 12. This is in conjunction with the physical situation since heat-conductivity decreases in the fluid, resulting in a reduction in thermal border thickening with increasing prandtl numbers.

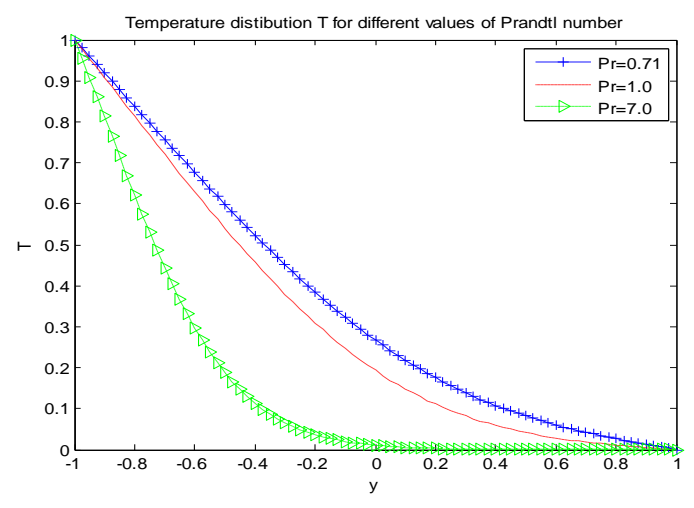

Fig.12. Impact of Prandtl Number on Temperature

\section{CONCLUSION}

There has been an explicit finite difference scheme used. The flow parameters investigated have been found to lead to the increase or decrease in velocity profiles and temperature distribution as follows; with an improvement in Darcy and Grashof numbers, primary velocity increases. However, with an improvement in suction and Prandtl estimates, it decreased. With an improvement in the amount of Darcy, Grashof and Suction parameters, secondary velocity increases. Secondary reductions with a rise in the number of Prandtl. With the increase in Grashof number, the temperature rises and with the enlargement in Prandtl number and suction number, it decreases. By increasing the Darcy number, there is a limited temperature effect.

\section{REFERENCES}

[1] Abuga J.K, Kinyanjui M and Sigey J.K,"An investigation of the effect of hall current and rotational parameter on dissipative fluid flow past a vertical semi-infinite plate", Journal of Engineering and Technology Research Vol. 3(11), pp. 314-320, October 2011.
[2] Acharya M, Dash G.C and Singh L.P,'”Magnetic field effects on the Free Convection and mass transfer flow through porous medium with constant suction and constant heat flux", Indian Journal of Pure and Applied Mathematics 31(1), pp:1-18, 2000 .

[3] Ahammad M.U, Obayedullah Md. and Rahman M.M, "Analysis of MHD free convection flow along a vertical porous plate embedded in a porous medium with magnetic field and heat generation", Journal of Engineering e-transaction 8(1), pp:10-18,2013.

[4] Ahmmed S.F, Ahmed R and Ali L. E, "Numerical study of unsteady free convection and mass transfer flow past a vertical porous plate", International journal of innovative research in advanced engineering 2(2), pp:278-285,2015.

[5] Ariful I, Muhammad M.I, MahaburL, Lasker E. A and Md. Shaklaoath K, "Unsteady Heat Transfer of Viscous Incompressible Boundary Layer Fluid flow through a Porous Plate with Induced Magnetic Field”, Journal of Applied Mathematics and Physics vol 4, pp:294-306, 2016.

[6] Atul K. S, Ajay K. S and N. P Singh,"Hydro magnetic free convection and mass transfer flow with Joule Heating, Thermal Diffusion, Heat Source and Hall Current," Bulletin of the Institute of Mathematics Academia Sinica 33(3), pp: 291-310, 2005.

[7] Jabeen K, Mushtaq M and Akram R.M,"Analysis of the MHD boundary layer flow over a nonlinear stretching sheet in a porous medium using semi analytical approaches", Hindawi Journal of Mathematical problems in engineering, pp: 1-9, 2020.

[8] Loganathan P and Sivapoornapriya C,'Unsteady natural convective flow over an impulsively started semi-infinite vertical plate in the presence of porous medium with chemical reaction", Journal of Applied fluid Mechanics 9(1), pp: 95-102, 2016.

[9] Laisal M, Mohammad S. H, Sanjitkumar P and Rubindra N. M, "MHD free convection heat and mass transfer flow over a vertical porous plate in a rotating system with hall current, heat source and suction", International journal of advances in applied mathematics and mechanics,6(1), pp:49-64,2018.

[10] Sreekath S, Nagarajan A.S and Venkata Ramana S,"Transient MHD free convection flow of an incompressible viscous dissipative fluid", Indian Journal of Pure and Applied Mathematics 32(7), pp: 1051-1058, 2001. 